\title{
Nonpositive Curvature and the Ptolemy Inequality
}

\author{
Thomas Foertsch $^{1}$, Alexander Lytchak ${ }^{1}$, Viktor Schroeder $^{2}$ \\ ${ }^{1}$ Universität Bonn, Mathematisches Institut, Beringstr. 1, 53115 Bonn, \\ Germany, and ${ }^{2}$ Universität Zürich, Institut für Mathematik, \\ Winterthurerstr. 190, 8057 Zürich, Switzerland
}

Correspondence to be sent to: Thomas Foertsch, Universität Bonn, Mathematisches Institut, Beringstr. 1, 53115 Bonn, Germany.e-mail:foertsch@math.uni-bonn.de

We provide examples of nonlocally, compact, geodesic Ptolemy metric spaces which are not uniquely geodesic. On the other hand, we show that locally, compact, geodesic Ptolemy metric spaces are uniquely geodesic. Moreover, we prove that a metric space is CAT(0) if and only if it is Busemann convex and Ptolemy.

\section{Introduction}

A metric space $X$ is called a Ptolemy metric space, if the inequality

$$
|x y||u v| \leq|x u||y v|+|x v||y u|
$$

is satisfied for all $x, y, u, v \in X$.

Our interest in Ptolemy metric spaces originates from an analysis of boundaries of CAT(-1)-spaces when endowed with a Bourdon or Hamenstädt metric. Such boundaries are indeed Ptolemy metric spaces (see Ref. [8]).

Various aspects of such spaces have occasionally been studied, for instance in Refs [5], [11], [13] and [18]. A smooth Riemannian manifold is of nonpositive sectional

See http://www.oxfordjournals.org/our_journals/imrn/for proper citation instructions.

(C) The Author 2007. Published by Oxford University Press. All rights reserved. For permissions, please e-mail:journals.permissions@oxfordjournals.org. 
curvature, if and only if it is locally Ptolemy, and a locally Ptolemy Finsler manifold is necessarily Riemannian (see Refs [12] and [5]). Furthermore, CAT(0)-spaces are geodesic Ptolemy metric spaces (compare Section 2).

On first consideration, these observations might suggest that for geodesic metric spaces the Ptolemy condition is some kind of nonpositive curvature condition. We show that without any further conditions this is completely incorrect.

THEOREM 1.1. Let $X$ be an arbitrary Ptolemy space, then $X$ can be isometrically embedded into a complete geodesic Ptolemy space $\hat{X}$.

As an application, take the four point Ptolemy space $X=\left\{x, y, m_{1}, m_{2}\right\}$ with $|x y|=$ 2 and all other nontrivial distances equal to 1 . By Theorem $1.1 X$ can be isometrically embedded into a geodesic Ptolemy space $\hat{X}$. Since $m_{1}$ and $m_{2}$ are midpoints of $x$ and $y$, there are in $\hat{X}$ two different geodesics joining the points $x$ and $y$. In particular, the space is not uniquely geodesic and hence far away from "nonpositively curved".

The space $\hat{X}$ constructed in Theorem 1.1 fails to be proper. Indeed, for proper geodesic Ptolemy spaces the situation is completely different:

THEOREM 1.2. A proper, geodesic, Ptolemy metric space is uniquely geodesic.

The following open question arises naturally.

Question: Is a proper, geodesic Ptolemy metric space necessarily a CAT(0)space?

The proof of Theorem 1.2 also works if one replaces "properness" through the assumption that there exists a geodesic bicombing which varies continuously with its endpoints. This will help us to prove that the property of being Ptolemy precisely distinguishes between the two most common nonpositive curvature conditions for geodesic metric spaces, namely the one due to Alexandrov and the one due to Busemann.

THEOREM 1.3. A metric space is $\mathrm{CAT}(0)$ if and only if it is Ptolemy and Busemann convex.

We finish the introduction with a few comments on the theorems above.

The space $\hat{X}$ constructed in Theorem 1.1 has several remarkable properties. There is a huge collection of convex functions on $\hat{X}$, in particular, all distance functions to points are convex. However, from the geometrical or topological point of view the space appears rather odd. Starting with finite spaces, for instance with the four point space $X$ as above, one obtains a space $\hat{X}$ on which the distance functions $d_{X}$ to the points $x \in X \subset \hat{X}$ are affine. This is to our knowledge, the first example of very strange affine 
functions on metric spaces. The space $\hat{X}$ is far from being a product, in contrast to the structural results obtained in Refs. [16] and [10]. Theorem 1.1 shows that the Ptolemy condition is sufficient to ensure that a metric space can be isometrically embedded into a geodesic Ptolemy metric space. This seems to be particularly interesting, as the problem of synthetic descriptions of (nonconvex) subsets of CAT(0)-spaces is very difficult (cf. [9]). The properness, assumed in addition in Theorem 1.2, forces the space to be contractible; in fact an absolute neighborhood retract. Moreover, it can be shown that the distance functions to points are never affine in this case, in fact they are strictly convex (cf. Remark 4.1). The proof of Theorem 1.3 confirms the following idea. If a Busemann convex space is not $\operatorname{CAT}(0)$, then it contains an infinitesimal portion of a nonEuclidean Banach space. This observation may be of some interest in its own right.

Finally, we want to draw the reader's attention to Ref. [2], a recent joint work of Berg and Nikolaev. In Ref. [2] the authors consider another four point condition on metric spaces, the so called quadrilateral condition, which one derives from the Ptolemy inequality by replacing the products of distances through the sums of their squares. Especially in the light of our Theorem 1.1, it seems remarkable to us, that such a variant of the Ptolemy inequality indeed forces a geodesic space to be CAT(0).

After a preliminary section, we prove Theorem 1.1 in Section 3, Theorem 1.2 in Section 4 and Theorem 1.3 in Section 5.

\section{Preliminaries}

We start by recalling some easy examples. The real line $\mathbb{R}$ is a Ptolemy space. To show this, consider points $x, y, z, w$ in this order on the line. A completely trivial computation shows that $|x z||y w|=|x y||z w|+|y z||w x|$, which implies that $\mathbb{R}$ is Ptolemy. Since the Ptolemy condition on four points is Möbius invariant (see below), the equality above holds for points $x, y, z, W$, which lie in this order on a circle in the plane $\mathbb{R}^{2}$. This is the classical Theorem of Ptolemy for cyclic quadrilaterals.

To show that the Euclidean space $\mathbb{R}^{n}$ is Ptolemy, consider again four points $x, y, z, w$. Applying a suitable Möbius transformation we can assume that $z$ is a midpoint of $y$ and $w$, that is $|y z|=|z w|=\frac{1}{2}|y w|$. For this configuration the Ptolemy inequality is equivalent to $|x z| \leq \frac{1}{2}(|x y|+|x w|)$, which is just the convexity of the distance to the point $x$.

Every CAT(0)-space is Ptolemy, since every four point configuration in a CAT(0)space admits a subembedding into the Euclidean plane ([4], p. 164). 
Möbius invariance: Let $d, d^{\prime}$ be two metrics on the same set $X$. The metrics are called Möbius equivalent, if for all quadruples $x, y, z, w$ of points

$$
\frac{d(x, y) d(z, w)}{d(x, z) d(y, w)}=\frac{d^{\prime}(x, y) d^{\prime}(z, w)}{d^{\prime}(x, z) d^{\prime}(y, w)} .
$$

If $d$ and $d^{\prime}$ are Möbius equivalent, then $(X, d)$ is Ptolemy, if and only if $\left(X, d^{\prime}\right)$ is Ptolemy.

Indeed, the Ptolemy inequality says that for all quadruples of points the three numbers of the triple

$$
A=(d(x, y) d(z, w), d(x, z) d(y, w), d(x, w) d(y, z))
$$

satisfy the triangle inequality. By dividing all three numbers by $d(x, w) d(y, z)$, we see that these numbers satisfy the triangle inequality if and only if the three numbers of the triple

$$
B=\left(\frac{d(x, y) d(z, w)}{d(x, w) d(y, z)}, \frac{d(x, z) d(y, w)}{d(x, w) d(y, z)}, 1\right)
$$

satisfy the triangle inequality. In this expression we can replace $d$ by $d^{\prime}$, and hence we obtain the claim.

Basic properties of Ptolemy spaces: Here we can view a couple of basic properties of Ptolemy spaces which will be frequently used in the remainder of this article.

(P1) Every subset $Y \subset X$ of a Ptolemy metric space $X$, endowed with the metric inherited from $X$, is Ptolemy.

(P2) A metric space $X$ is Ptolemy if and only if for every $\lambda>0$ the scaled space $\lambda X$ is Ptolemy.

Some of our arguments below will use the notions of ultrafilters and ultralimits; a generalization of pointed Gromov-Hausdorff convergence. We refer the reader, not familiar with these methods, to Refs. [4] and [15]. The symbol $\lim _{\omega}\left(X_{n}, X_{n}\right)$ will denote such an ultralimit (w.r.t. a nonprincipal ultrafilter $\omega$ ).

As every metric property, the Ptolemy condition is invariant w.r.t. ultraconvergence.

(P3) For every sequence $\left\{\left(X_{i}, X_{i}\right)\right\}_{i}$ of pointed Ptolemy spaces and every nonprinciple ultrafilter $\omega$, the ultralimit $\lim _{\omega}\left(X_{i}, X_{i}\right)$ is a Ptolemy space.

Furthermore, we recall another important observation, which is due to Schoenberg (see Ref. [19]). This property lies in the heart of Theorem 1.3. 
(P4) A normed vector space is an inner product space if and only if it is Ptolemy.

A subset of a normed vector space is called linearly convex, if with any two points it contains the straight line segment connecting these points. A metric space is called linearly convex, if it is isometric to a linearly convex subset of a normed vector space and called flat, if it is isometric to a convex subset of an inner product space.

With this notation the properties above immediately yield the following corollary.

COROLlaRY 2.1. Let $X$ be a Ptolemy space, then every linearly convex subset $C \subset X$ of $X$ is flat.

Let $X$ be a metric space and $X, y \in X$. Then a point $m \in X$ is called a midpoint of $x$ and $y$ if $|x m|=\frac{1}{2}|x y|=|m y|$. We say that $X$ has the midpoint property, if for all $x, y \in X$ there exists a midpoint of $x$ and $y$ in $X$. The space $X$ is called geodesic, if any two points $X, y \in X$ can be joined by a geodesic path, that is, to any two points $X, y \in X$ there exists an isometric embedding $\gamma$ of the interval $[0,|x y|]$ of the Euclidean line into $X$ such that $\gamma(0)=x$ and $\gamma(|x y|)=y$. In this article we will always assume that geodesics are parameterized affinely, that is, proportionally to arclength. A complete space with the midpoint property is geodesic. Note that a Ptolemy metric space $X$ which has the midpoint property satisfies

$$
|m z| \leq \frac{1}{2}[|x z|+|y z|]
$$

for all $x, y, z, m \in X$ such that $m$ is a midpoint of $x$ and $y$ (cf. [7] for a discussion of such spaces).

Inequality equation (2.1) implies a further notable property of geodesic Ptolemy metric spaces, namely

(P5) In a geodesic Ptolemy metric space distance functions to points are convex.

Nonpositive curvature conditions and often convex spaces: The most common nonpositive curvature conditions are due to Alexandrov and Busemann (cf. [1] and [6]).

We suppose that the reader interested in this article's subject is familiar with the notion of CAT(0)-spaces. Roughly speaking, a geodesic space $X$ is called a CAT(0)space if all geodesic triangles in $X$ are not thicker than their comparison triangles in the Euclidean plane $\mathbb{E}^{2}$ (for a precise definition we refer the reader, to Section II.1 in Ref. [4]).

The geodesic space $X$ is said to be Busemann convex, if for any two (affinely parameterized) geodesics $\alpha, \beta: I \longrightarrow X$, the map $t \mapsto|\alpha(t) \beta(t)|$ is convex. 
REMARK 2.2. Every CAT(0)-space is Busemann convex and every Busemann convex space is uniquely geodesic. However, there are Busemann convex spaces which are not CAT(0)spaces, as for instance all non-Euclidean normed vector spaces with strictly convex unit norm balls.

The notion of Busemann convexity is not stable under limit operations. For instance, a sequence of strictly convex norms on $\mathbb{R}^{n}$ may converge to a nonstrictly convex norm. To overcome such a phenomenon, Bruce Kleiner introduced a weaker notion of Busemann convexity in Ref. [14], which is stable with respect to limit operations. A metric space $X$ is called often convex, if there exists a convex geodesic bicombing, that is, a map $\gamma: X \times X \times[0,1] \rightarrow X,(X, Y, t) \mapsto \gamma_{X, Y}(t)$, such that $t \mapsto \gamma_{X, Y}(t)$ is a geodesic with $\gamma_{X, Y}(0)=x, \gamma_{X, Y}(1)=y$, and for all $x, y, x^{\prime}, y^{\prime}$ and all (not necessarily surjective) affine maps $\varphi: I \rightarrow[0,1], \psi: I \rightarrow[0,1]$ defined on the same interval $I$ the map $s \mapsto$ $\left|\gamma_{X, Y}(\varphi(s)) \gamma_{X^{\prime}, Y^{\prime}}(\psi(s))\right|$ is convex. This convexity implies in particular, that $\gamma$ is continuous and that for points $x^{\prime}, y^{\prime} \in \gamma_{X, Y}([0,1])$ the geodesic $\gamma_{x^{\prime}, Y^{\prime}}$ is contained in $\gamma_{X, Y}$. In particular, $\gamma$ defines a continuous midpoint map $m(x, y):=\gamma_{x, y}(1 / 2)$.

We state the following properties of often convex spaces, that are direct consequences of the definitions (cf. [14]).

(OC1) For every sequence $\left\{\left(X_{i}, X_{i}\right)\right\}_{i}$ of often convex spaces and every nonprinciple ultrafilter $\omega$, the ultralimit $\lim _{\omega}\left(X_{i}, X_{i}\right)$ is an often convex space.

(OC2) A metric space $X$ is often convex if and only if for every $\lambda>0$ the scaled space $\lambda X$ is often convex.

(OC3) Let $X$ be often convex. Then $X$ is Busemann convex if and only if $X$ is uniquely geodesic.

\section{Proof of Theorem 1.1}

We explicitly construct the complete geodesic Ptolemy metric space $\hat{X}$. First, we subsequently add midpoints to $X$ in order to obtain a Ptolemy metric space $\mathfrak{M}(X)$ which has the midpoint property. Then we pass to an ultraproduct of $\mathfrak{M}(X)$.

Let $\Sigma$ denote the set of unordered tuples in $X$. Formally, $\Sigma=\left\{\left\{x_{1}, x_{2}\right\} \subset X \mid x_{1}, X_{2} \in\right.$ $X\}$, that is $\Sigma$ consists of all subsets of $X$ with one or two elements.

On $\Sigma$ we define a metric via

$$
\left|\left\{x_{1}, x_{2}\right\}\left\{y_{1}, y_{2}\right\}\right|:=\left\{\begin{array}{cl}
\frac{1}{4}\left[\left|x_{1} y_{1}\right|+\left|x_{1} y_{2}\right|+\left|x_{2} y_{1}\right|+\left|x_{2} y_{2}\right|\right] & \text { if }\left\{x_{1}, y_{1}\right\} \neq\left\{y_{1}, y_{2}\right\} \\
0 & \text { if }\left\{x_{1}, x_{2}\right\}=\left\{y_{1}, y_{2}\right\}
\end{array}\right\}
$$


for all $\left\{x_{1}, x_{2}\right\},\left\{y_{1}, y_{2}\right\} \in \Sigma$. This indeed defines a metric on $\Sigma$. In order to verify this, one has to prove the triangle inequality

$$
\left|\left\{x_{1}, x_{2}\right\}\left\{y_{1}, y_{2}\right\}\right| \leq\left|\left\{x_{1}, x_{2}\right\}\left\{z_{1}, z_{2}\right\}\right|+\left|\left\{z_{1}, z_{2}\right\}\left\{y_{1}, y_{2}\right\}\right|
$$

for all $\left\{x_{1}, x_{2}\right\},\left\{y_{1}, y_{2}\right\},\left\{z_{1}, z_{2}\right\} \in \Sigma$. If two of the tuples coincide, the validity of the inequality above is evident, and otherwise it just follows by applying the triangle inequality in $X$ three times.

Moreover, the space $M(X):=(\Sigma,|\cdot|)$ is Ptolemy, that is, it satisfies

$$
\begin{aligned}
\left|\left\{x_{1}, x_{2}\right\}\left\{y_{1}, y_{2}\right\}\right| \cdot\left|\left\{z_{1}, z_{2}\right\}\left\{u_{1}, u_{2}\right\}\right| \leq & \left|\left\{x_{1}, x_{2}\right\}\left\{z_{1}, z_{2}\right\}\right| \cdot\left|\left\{y_{1}, y_{2}\right\}\left\{u_{1}, u_{2}\right\}\right| \\
& +\left|\left\{x_{1}, x_{2}\right\}\left\{u_{1}, u_{2}\right\}\right| \cdot\left|\left\{y_{1}, y_{2}\right\}\left\{z_{1}, z_{2}\right\}\right|
\end{aligned}
$$

for all $\left\{x_{1}, x_{2}\right\},\left\{y_{1}, y_{2}\right\},\left\{z_{1}, z_{2}\right\},\left\{u_{1}, u_{2}\right\} \in \Sigma$. Once again, the validity of this inequality is evident, if two of the tuples coincide, and otherwise it follows by applying the Ptolemy inequality in $X$ sixteen times.

Note further that $X$ isometrically embeds into $M(X)$ via $X \mapsto\{X, X\}$. Thus we may identify $X$ with a subset of $M(X)$.

Now we define $M^{0}(X):=X$ as well as $M^{k+1}(X):=M\left(M^{k}(X)\right)$ and set $\mathfrak{M}(X):=$

$\bigcup_{k=0}^{\infty} M^{k}(X)$. From the considerations above it follows that this space is a Ptolemy metric space. Moreover, it has the midpoint property. Namely, each pair $X, y \in \mathfrak{M}(X)$ is contained in some $M^{k}(X)$ and $\{x, y\} \in M^{k+1}(X)$ is a midpoint of $x$ and $y$. Passing to an ultraproduct $\hat{X}$ of $\mathfrak{M}(X)$, that is, $\hat{X}:=\lim _{\omega}\{(\mathfrak{M}(X), \mathfrak{x})\}_{n}$, the ultralimit of the constant sequence $\{(\mathfrak{M}(X), \mathfrak{x})\}_{n}$ w.r.t. some ultrafilter $\omega$, where $\mathfrak{x} \in \mathfrak{M}(X)$, we obtain a complete Ptolemy metric space which has the midpoint property and therefore, is geodesic.

\section{Proof of Theorem 1.2}

In this section we prove Theorem 1.2. For two points $p^{-}, p^{+} \in X$ we consider the set

$$
C\left(p^{-}, p^{+}\right):=\left\{x \in X|| p^{-} p^{+}|=| p^{-} x|+| x p^{+} \mid\right\}
$$

Since the distance functions $d_{p^{ \pm}}:=d\left(p^{ \pm}, \cdot\right): X \rightarrow \mathbb{R}_{0}^{+}$are convex, the set $C\left(p^{-}, p^{+}\right)$is convex. The convexity of $d_{p^{ \pm}}$implies that these functions are affine on the set $C\left(p^{-}, p^{+}\right)$.

PROOF OF THEOREM 1.2. Let $\gamma_{1}, \gamma_{2}:\left[0, L:=\left|p^{-} p^{+}\right|\right] \rightarrow C$ be geodesics connecting $p^{-}$to $p^{+}$. Set $x_{s}:=\gamma_{1}(s)$ as well as $y_{s}:=\gamma_{2}(s)$ and let $m_{s}$ denote a midpoint of $x_{s}$ and $y_{s}$. Since the 


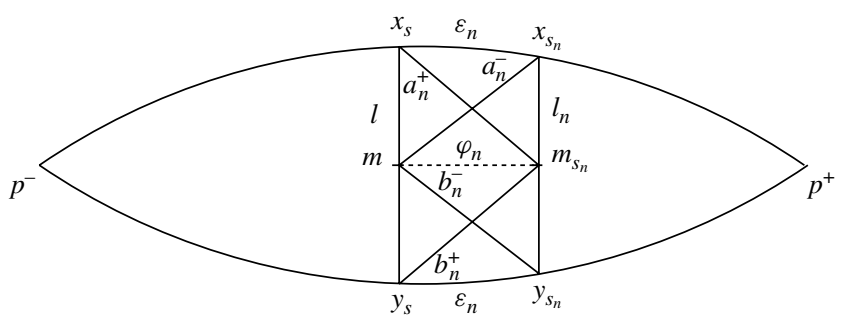

Fig. 1 This figure visualizes the notation used in the proof.

functions $d_{p^{ \pm}}: C\left(p^{-}, p^{+}\right) \rightarrow \mathbb{R}_{0}^{+}$are affine, we obtain $\left|p^{-} m_{s}\right|=s$ and $\left|p^{+} m_{s}\right|=L-s$. (See Figure 1.)

Let now $0<s<t \leq L$ be arbitrary, then the triangle inequality yields

$$
\left|m_{s} x_{t}\right|+\left|m_{s} y_{t}\right| \geq\left|x_{t} Y_{t}\right|
$$

the Ptolemy inequality yields

$$
\left|p^{-} m_{t}\right| \cdot\left|x_{S} Y_{s}\right| \leq\left|m_{t} X_{s}\right| \cdot\left|p^{-} y_{s}\right|+\left|m_{t} Y_{s}\right| \cdot\left|p^{-} x_{s}\right|
$$

and therefore

$$
\left|m_{t} X_{s}\right|+\left|m_{t} Y_{s}\right| \geq \frac{t}{s} \cdot\left|X_{S} Y_{s}\right|=\left|X_{s} Y_{s}\right|+(t-s) \frac{\left|X_{s} Y_{s}\right|}{s} .
$$

On the other hand the Ptolemy property for the points $m_{s}, x_{t}, m_{t}, x_{s}$ and $m_{s}, y_{t}, m_{t}, Y_{s}$ yields

$$
\left|m_{s} X_{t}\right| \cdot\left|m_{t} X_{s}\right|+\left|m_{s} Y_{t}\right| \cdot\left|m_{t} Y_{s}\right| \leq \frac{1}{2}\left|x_{s} Y_{s}\right| \cdot\left|x_{t} y_{t}\right|+2(t-s) \cdot\left|m_{s} m_{t}\right| .
$$

Now, fix any $0<s<L$, set $l:=\left|x_{S} Y_{s}\right| \geq 0$, and choose a sequence $s_{n} \rightarrow s$ with $s_{n}>s$. Let $m_{s_{n}}$ denote midpoints of $x_{s_{n}}$ and $Y_{s_{n}}$. By compactness, we can pass to a subsequence $m_{s_{n}} \rightarrow m$, where $m=m_{s}$ is a midpoint of $x_{s}$ and $y_{s}$. Now, we set

$$
\begin{aligned}
& \varphi_{n}:=\left|m_{s_{n}} m\right|, \quad \epsilon_{n}:=s_{n}-s, \quad a_{n}^{+}:=\left|m_{s_{n}} x_{s}\right|, \quad b_{n}^{+}:=\left|m_{s_{n}} y_{s}\right| \\
& a_{n}^{-}:=\left|m, X_{s_{n}}\right|, \quad b_{n}^{-}:=\left|m Y_{s_{n}}\right|, \quad l_{n}:=\left|X_{S_{n}} Y_{s_{n}}\right| \quad \text { and } \quad Q:=\frac{l}{s} \geq 0 \text {. }
\end{aligned}
$$

We have by Equations (4.1), (4.2) and (4.3):

$$
a_{n}^{-}+b_{n}^{-} \geq l_{n}, \quad a_{n}^{+}+b_{n}^{+} \geq l+\epsilon_{n} \cdot Q, \quad b_{n}^{+} \cdot b_{n}^{-}+a_{n}^{+} \cdot a_{n}^{-} \leq \frac{1}{2} l \cdot l_{n}+2 \epsilon_{n} \varphi_{n} .
$$


By triangle inequalities, we see $\left|l_{n}-l\right| \leq 2 \epsilon_{n},\left|a_{n}^{ \pm}-\frac{1}{2} l\right| \leq 2 \epsilon_{n},\left|b_{n}^{ \pm}-\frac{1}{2} l\right| \leq 2 \epsilon_{n}$, hence passing to a subsequence, we find $A^{+}, A^{-}, B^{+}, B^{-}, C \in[-2,2]$ such that $a_{n}^{ \pm}=$ $\frac{1}{2} l+A^{ \pm} \epsilon_{n}+o\left(\epsilon_{n}\right), b_{n}^{ \pm}=\frac{1}{2} l+B^{ \pm} \epsilon_{n}+o\left(\epsilon_{n}\right)$ and $l_{n}=l+C \epsilon_{n}+o\left(\epsilon_{n}\right)$. Since $2 \epsilon_{n} \varphi_{n}=o\left(\epsilon_{n}\right)$, we derive from (4.4) that

$$
B^{-}+A^{-} \geq C, \quad B^{+}+A^{+} \geq Q \geq 0, \quad\left(B^{+}+B^{-}\right)+\left(A^{+}+A^{-}\right) \leq C .
$$

Thus $Q=0$, which implies $\gamma_{1}(s)=\gamma_{2}(s)$. Since $s \in(0, L)$ was arbitrary, we have $\gamma_{1}=\gamma_{2}$.

REMARK 4.1. It is not difficult to prove that in Theorem 1.2 one can replace "properness" by the slightly weaker assumption of "local compactness". A more involved argument shows that a locally compact geodesic Ptolemy metric space is even strictly distance convex, that is, that the inequality equation (2.1) is strict, whenever $|x y|>|| x z|-| z y \|$. The proof of this claim will be given elsewhere.

We finish this section with an important observation. In the proof of Theorem 1.2 we use the properness of $X$ only to show that $m_{s_{n}} \rightarrow m$. Clearly, the existence of a continuous midpoint map $m: X \times X \rightarrow X$ also implies this convergence. As a consequence we have the following:

THEOREM 4.2. Let $X$ be a geodesic, Ptolemy space which admits a continuous midpoint map. Then $X$ is uniquely geodesic.

Since often convex spaces admit continuous midpoint maps, the first statement of the following corollary is an immediate consequence.

COROLlary 4.3. Let $X$ be an often convex and Ptolemy space. Then $X$ is uniquely geodesic and hence, by (OC3), Busemann convex. Moreover, for every sequence of pointed Busemann convex and Ptolemy metric spaces $\left\{\left(X_{i}, x_{i}\right)\right\}$, every nonprinciple ultrafilter $\omega$ and all $\lambda_{i} \in \mathbb{R}^{+}$, the ultralimit $\lim _{\omega}\left(\lambda_{i} X_{i}, x_{i}\right)$ is Busemann convex.

The second statement goes as follows: A Busemann convex space is in particular often convex. By (OC1) this property and by (P3) the Ptolemy property is stable under limits. Thus the limit is often convex and Ptolemy and hence Busemann convex.

\section{Proof of Theorem 1.3}

In this section we prove Theorem 1.3. First, we recall the notion of generalized and weak angles (cf. Ref. [15]). Then we prove Proposition 5.3 (a version of the Toponogov rigidity 
theorem), that was shown independently by Rinow and Bowditch under slightly more restrictive conditions (see Refs [17] and [3]), and which will finally allow us to obtain a proof of our Theorem 1.3.

\subsection{Ultrarays associated to geodesics and their enclosed angles}

Ultrarays: Let $X$ be a geodesic metric space and let $\gamma$ be a geodesic in $X$ emanating from $p \in X$. Now take a nonprinciple ultrafilter $\omega$, consider the $\omega$-blow up $(\bar{X}, \bar{d})$ of $X$ in $p$, that is $(\bar{X}, \bar{d}):=\lim _{\omega}\{(n X, p)\}_{n}$, and define $\bar{\gamma}:[0, \infty) \longrightarrow \bar{X}$ through $\bar{\gamma}(s):=\lim _{\omega}\left\{\left(\gamma\left(\frac{s}{n}\right), p\right)\right\}_{n}$ for all $s \in[0, \infty)$. This map is indeed a geodesic ray in $(\bar{X}, \bar{d})$ emanating in $\{p\}_{n} \in \bar{X}$. We call $\bar{\gamma}$ the ultraray associated to $\gamma($ and $\omega)$.

Weak angles: In order to get a grip on the interplay between geodesics and their associated ultrarays, we recall certain notions of angles.

Given three points $p, x$ and $y$ in a metric space $X$, consider corresponding comparison points $p^{\prime}, x^{\prime}$ and $y^{\prime}$ in the Euclidean plane $\mathbb{E}^{2}$. Let $\left[p^{\prime}, x^{\prime}\right]$ and $\left[p^{\prime}, y^{\prime}\right]$ denote the geodesic segments in $\mathbb{E}^{2}$ connecting $p^{\prime}$ to $x^{\prime}$ and $p^{\prime}$ to $y^{\prime}$. These segments enclose an angle in $p^{\prime}$ and this angle is referred to as the (Euclidean) comparison angle of $x$ and $y$ at $p$. We write $\angle_{p}(x, y)$ for this angle.

Let now $X$ be a metric space and consider two geodesic segments $\gamma_{1}$ and $\gamma_{2}$ parameterized by arclength, both initiating in some $p \in X$. Then $\gamma_{1}$ and $\gamma_{2}$ are said to enclose the angle $\angle_{p}\left(\gamma_{1}, \gamma_{2}\right)$ (in the strict sense) at $p$ if the limit $\angle_{p}\left(\gamma_{1}, \gamma_{2}\right):=$ $\lim _{s, t \rightarrow 0} \angle_{p}\left(\gamma_{1}(s), \gamma_{2}(t)\right)$ exists.

Recall that a normed vector space is an inner product space if and only if all straight line segments emanating from the origin enclose an angle. However, even in normed vector spaces that are not inner product spaces certain so-called generalized angles do exist between any straight line segments initiating in a common point. Such generalized angles were introduced in Ref. [15].

Let $a, b>0$ and $\gamma_{1}$ and $\gamma_{2}$ be as above. then we say that $\gamma_{1}$ and $\gamma_{2}$ enclose a generalized angle $\angle_{p}^{g}\left(\gamma_{1}, a, \gamma_{2}, b\right)$ at scale $(a, b)$, if the limit

$$
\angle_{p}^{g}\left(\gamma_{1}, a, \gamma_{2}, b\right):=\lim _{s \rightarrow 0} \angle_{p}\left(\gamma_{1}(a s), \gamma_{2}(b s)\right)
$$

exists. If $\gamma_{1}$ and $\gamma_{2}$ enclose generalized angles at all scales $(a, b)$ and, moreover, these generalized angles do not depend on the particular scale, then we say that $\gamma_{1}$ and $\gamma_{2}$ 
enclose the weak angle

$$
\angle_{p}^{W}\left(\gamma_{1}, \gamma_{2}\right):=\angle_{p}^{g}\left(\gamma_{1}, 1, \gamma_{2}, 1\right)
$$

Ultrarays in Busemann convex spaces: Now let $X$ be Busemann convex and $\gamma_{1}$ and $\gamma_{2}$ be geodesics on $X$ with $\gamma_{1}(0)=p=\gamma_{2}(0)$. Then, for all scales $(a, b)$, the generalized angle $\angle_{p}^{g}\left(\gamma_{1}, a, \gamma_{2}, b\right)$ exists. This is immediate, since $s \mapsto \frac{\left|\gamma_{1}(a s) \gamma_{2}(b s)\right|}{s}$ is monotonously increasing.

Next consider the ultrarays $\overline{\gamma_{1}}$ and $\overline{\gamma_{2}}$ associated to $\gamma_{1}$ and $\gamma_{2}$. These ultrarays satisfy

$$
\bar{d}\left(\overline{\gamma_{1}}(a s), \overline{\gamma_{2}}(b s)\right)=s \cdot \bar{d}\left(\overline{\gamma_{1}}(a), \overline{\gamma_{2}}(b)\right) \quad \forall a, b, s>0
$$

Moreover, the existence of weak angles of geodesics $\gamma_{1}$ and $\gamma_{2}$ in a Busemann convex space is equivalent to the existence of angles (in the strict sense) between their associated ultrarays $\overline{\gamma_{1}}$ and $\overline{\gamma_{2}}$ in $\bar{X}$.

LEMMA 5.1. Let $X$ be Busemann convex, let $\gamma_{1}$ and $\gamma_{2}$ denote geodesics in $X$ initiating in a common point $p \in X$ and let $\overline{\gamma_{1}}$ and $\overline{\gamma_{2}}$ denote their associated ultrarays. Then the following properties are mutually equivalent.

(1) The rays $\gamma_{1}$ and $\gamma_{2}$ enclose a weak angle.

(2) The ultrarays $\overline{\gamma_{1}}$ and $\overline{\gamma_{2}}$ enclose an angle (in the strict sense).

(3) The union $\overline{\gamma_{1}}\left(\mathbb{R}^{+}\right) \cup \overline{\gamma_{2}}\left(\mathbb{R}^{+}\right)$admits an isometric embedding into the Euclidean plane $\mathbb{E}^{2}$.

PROOF. The equivalence of conditions (2) and (3) follows immediately from Equation (5.1). Moreover, this equation also implies that the ultrarays $\overline{\gamma_{1}}$ and $\overline{\gamma_{2}}$ enclose an angle if and only if they enclose a weak angle. Hence the equivalence of conditions (1) and (2) is a consequence of $\frac{1}{s} \bar{d}\left(\overline{\gamma_{1}}(a s), \overline{\gamma_{2}}(b s)\right)=\lim _{\omega}\left\{n\left|\gamma_{1}(a / n) \gamma_{2}(b / n)\right|\right\}_{n}$, and the fact that the generalized angles between $\gamma_{1}$ and $\gamma_{2}$ exist for all scales in any case.

Proposition 5.2. Let $X$ be Busemann convex. Assume that for all geodesic segments $\gamma_{1}$ and $\gamma_{2}$ with $\gamma_{1}(0)=p=\gamma_{2}(0)$, the weak angle $\angle_{p}^{W}\left(\gamma_{1}, \gamma_{2}\right)$ exists. Then $X$ is a CAT $(0)$-space.

PROOF. We first show that the weak angle satisfies the four axioms of an angle as formulated in Ref. [4] II.1.8 (p. 162). Thus, we have to show 
(A1) $\angle_{p}^{W}\left(\gamma_{1}, \gamma_{2}\right)=\angle_{p}^{W}\left(\gamma_{2}, \gamma_{1}\right)$

(A2) $\angle_{p}^{W}\left(\gamma_{1}, \gamma_{3}\right) \leq \angle_{p}^{W}\left(\gamma_{1}, \gamma_{2}\right)+\angle_{p}^{W}\left(\gamma_{2}, \gamma_{3}\right)$

(A3) if $\gamma_{2}$ is the restriction of $\gamma_{1}$ to an initial segment, then $\angle_{p}^{W}\left(\gamma_{1}, \gamma_{2}\right)=0$

(A4) if the concatenation of $\gamma_{1}=[p, x]$ and $\gamma_{2}=[p, y]$ is a geodesic $[x, y]$, then $\angle_{p}^{W}\left(\gamma_{1}, \gamma_{2}\right)=\pi$

Now (A1), (A3), (A4) are trivially true by the definition. Since weak angles between rays coincide (by Lemma 5.1) with the angles of their associated ultrarays and since such angles satisfy the triangle inequality also (A2) holds. Furthermore we see that $\angle_{p}^{W}([p, x],[p, y]) \leq \angle_{p}(x, y)$, which follows from the Busemann convexity. Now implication $(4) \Longrightarrow(2)$ on p.161 of II.1.7 in Ref. [4] remains valid, if one replaces the Alexandrov angle in condition (4) through the weak angle (cf. the paragraph after II.1.8 in Ref. [4]). This implies that $X$ is a $\operatorname{CAT}(0)$-space.

\subsection{A convex hull proposition}

The purpose of this subsection is to prove the following proposition.

PROPOSITION 5.3. (Compare [17], p. 432, par. 7 and p. 463, par. 20 as well as [3], Lemma 1.1. and the remark after its proof) Let $X$ be Busemann convex and let $\gamma_{1}, \gamma_{2}$ : $I \longrightarrow X$ be two linearly reparameterized (finite or infinite) geodesics in $X$ such that $t \mapsto\left|\gamma_{1}(t) \gamma_{2}(t)\right|$ is affine. Then the convex hull $C$ of $\gamma_{1}$ and $\gamma_{2}$ is a convex subset of a twodimensional normed vector space.

Given a geodesic metric space $X$, a function $f: X \longrightarrow \mathbb{R}$ is called affine if its restriction to each affinely parameterized geodesic $\gamma$ in $X$ satisfies $f(\gamma(t))=a t+b$ for some numbers $a, b \in \mathbb{R}$ that may depend on $\gamma$. We say that affine functions on $X$ separate points, if for each pair of distinct points $X, X^{\prime} \in X$ there is an affine function $f: X \longrightarrow \mathbb{R}$ with $f(x) \neq f\left(x^{\prime}\right)$. With this terminology the following theorem has been proven in Ref. [10].

THEOREM 5.4. (Theorem 1.1 in [10]) Let $X$ be a geodesic metric space. If affine functions on $X$ separate points then $X$ is isometric to a convex subset of a normed vector space with a strictly convex norm.

Using this result, we are able to provide the proof.

PROOF OF PROPOSITION 5.3. Let $y_{t}: I_{t} \longrightarrow X$ be the geodesic from $\gamma_{1}(t)$ to $\gamma_{2}(t)$ where $I_{t}=\left[0,\left|\gamma_{1}(t) \gamma_{2}(t)\right|\right]$. Let $C_{0}:=\bigcup_{t \in I} Y_{t}\left(I_{t}\right)$. 


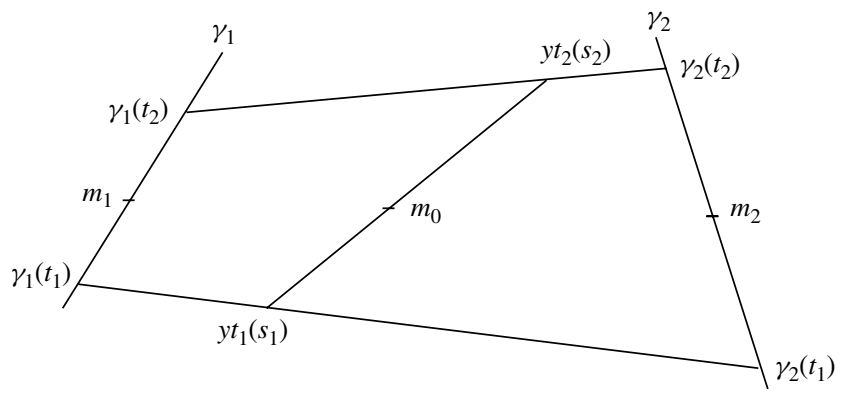

Fig. 2 This figure visualizes our notation used in the proof of Proposition 5.3.

Claim: $C_{0}$ is convex, that is, $C_{0}=C$.

In order to prove this claim, we may assume that $\gamma_{1}$ and $\gamma_{2}$ are closed. Then $C_{0}$ is closed and it is sufficient to prove that for $t_{1}, t_{2} \in I, s_{1} \in I_{t_{1}}$ and $s_{2} \in I_{t_{2}}$ the midpoint of $y_{t_{1}}\left(s_{1}\right)$ and $y_{t_{2}}\left(s_{2}\right)$ is contained in $C_{0}$. (See Figure 2.)

Let $m$ denote the unique midpoint map on $X$ and set

$$
m_{0}:=m\left(y t_{1}\left(s_{1}\right), y t_{2}\left(s_{2}\right)\right), m_{1}:=\gamma_{1}\left(\frac{t_{1}+t_{2}}{2}\right), \text { and } m_{2}:=\gamma_{2}\left(\frac{t_{1}+t_{2}}{2}\right) .
$$

From the convexity of the distance function and the fact that

$$
\left|m_{1} m_{2}\right|=\frac{1}{2}\left[\left|\gamma_{1}\left(t_{1}\right) \gamma_{2}\left(t_{1}\right)\right|+\left|\gamma_{1}\left(t_{2}\right) \gamma_{2}\left(t_{2}\right)\right|\right],
$$

we deduce that $\left|m_{1} m_{2}\right|=\left|m_{1} m_{0}\right|+\left|m_{0} m_{2}\right|$. Thus $m_{0}$ is contained in the geodesic $y \frac{t_{1}+t_{2}}{2}$. This proves the claim.

In fact, with the same reasoning as above we deduce more, namely that $\left|m_{0} m_{1}\right|=$ $\frac{s_{1}+s_{2}}{2}$. This shows that the function $F_{1}: C \longrightarrow \mathbb{R}$, given through $F_{1}\left(y_{t}(s)\right)=s$ is affine on $C$.

Moreover, the function $F_{2}: C \longrightarrow \mathbb{R}$, given through $F_{2}\left(y_{t}(s)\right)=t$ is affine as well, since the midpoint between $y_{t_{1}}\left(s_{1}\right)$ and $y_{t_{2}}\left(s_{2}\right)$ lies on the geodesic $Y \frac{t_{1}+t_{2}}{2}$.

Now the affine functions $F_{1}$ and $F_{2}$ separate the points of $C$. Thus we can apply Theorem 5.4, which finishes the proof.

\subsection{Proof of Theorem 1.3}

Every CAT(0)-space is both, Ptolemy and Busemann convex. It remains to show that a Busemann convex and Ptolemy metric space is already CAT(0). 
In order to reach a contradiction, suppose that $X$ is Ptolemy and Busemann convex but not CAT(0). Then, due to Proposition 5.2 there do exist two geodesics $\gamma_{1}$ and $\gamma_{2}$ that do not enclose a weak angle at their common starting point $p=\gamma_{1}(0)=\gamma_{2}(0)$. Let $\overline{\gamma_{1}}$ and $\overline{\gamma_{2}}$ denote the geodesic rays defined in $Y=\lim _{\omega}\{n X, p\}_{n}$ as above. Then, due to Lemma 5.1, $\overline{\gamma_{1}}$ and $\overline{\gamma_{2}}$ do not enclose an angle in $\{p\}_{n} \in Y$ either.

Now $Y$ is Busemann convex by Corollary 4.3. Moreover, the function $t \mapsto$ $\left|\overline{\gamma_{1}}(t) \overline{\gamma_{2}}(t)\right|$ is linear. Thus, by Proposition 5.3, the convex hull $C$ of $\overline{\gamma_{1}}$ and $\overline{\gamma_{2}}$ is isometric to a convex set of a two-dimensional normed vector space. Since $Y$ is Ptolemy, $C$ is flat by Corollary 2.1. It follows that $\overline{\gamma_{1}}$ and $\overline{\gamma_{2}}$ enclose an angle, which yields the desired contradiction.

\section{Acknowledgments}

The work by TF and AL was supported by Collaborative Research Center (SFB) 611. VS's work was supported by Swiss National Science Foundation.

\section{References}

[1] Alexandrov, A. D. "A Theorem on Triangles in a Metric Space and Some of its Applications." Trudy Mathematics Institution Steklov 38 (1951): 5-23.

[2] Berg, I. D., and I. G. Nikolaev. "Quasilinearization and Curvature of Aleksandrov Spaces." Doklady Mathematics 75, no. 3 (2007): 336-338.

[3] Bowditch, B. H. "Minkowskian Subspaces of Non-positively Curved Metric Spaces." Bulletin of the London Mathematical Society 27, no. 6 (1995): 575-584.

[4] Bridson, M., and A. Haefliger. Metric Spaces of Non-positive Curvature, Grundlehren der mathematischen Wissenschaften, vol. 319 (Berlin: Springer Verlag, 1999), xxii-643.

[5] Buckley, S. M., K., Falk and D. J. Wraith. "Ptolemaic Spaces and CAT(0)," forthcoming.

[6] Busemann, H. "Spaces with Non-positive Curvature." Acta Mathematica 80 (1948): 259-310.

[7] Foertsch, T. "Ball Versus Distance Convexity of Metric Spaces." Beiträge zur Algebra und Geometrie 45, no. 2 (2004): 481-500.

[8] Foertsch, T., and V. Schroeder. "Hyperbolicity, CAT(-1)-spaces and the Ptolemy Inequality," forthcoming.

[9] Gromov, M. “CAT $(\kappa)$-spaces: Construction and Concentration." Geometry i Topology 7, 280 (2001): 100-140, 299-300, translation in Journal of Mathematical Sciences 119, no. 2 (2004): 178-200.

[10] Hitzelberger, P., and A. Lytchak. "Spaces with many Affine Functions." Proceedings of the American Mathematical Society 135 (2007): 2263-2271. 
[11] Kay, D. C. "The Ptolemaic Inequality in Hilbert Geometries." Pacific Journal of Mathematics 21 (1967): 293-301.

[12] Kay, D. C. "Ptolemaic metric spaces and the characterization of geodesics by vanishing metric curvature", Michigan State University Doctoral Dissertation, Ann Arbor Microfilms, 1963.

[13] Klamkin, M. S., and A. Meir. "Ptolemy's Inequality, Chordal Metric, Multiplicative Metric." Pacific Journal of Mathematics 101 (1982): 389-392.

[14] Kleiner, B. "The Local Structure of Length Spaces with Curvature Bounded Above." Mathematische Zeitschrift 231 (1999): 409-456.

[15] Lytchak, A. "Differentiation in Metric Spaces." Algebra i Analiz 16, no. 6 (2004): 128-161, translation in St. Petersburg Mathematical Journal 16, no. 6 (2005): 1017-1041.

[16] Lytchak, A., and V. Schroeder. "Affine Functions on CAT $(\kappa)$-spaces." Mathematische Zeitschrift 255, no. 2 (2007): 231-244.

[17] Rinow, W. Die Innere Geometrie der Metrischen Räume, Die Grundlehren der mathematischen Wissenschaften, Bd. 105. (Berlin-Göttingen-Heidelberg: Springer-Verlag, 1961), xv-520.

[18] Smith, J. D. "Generalization of the Triangle and Ptolemy Inequalities." Geometriae Dedicata 50 (1994): 251-259.

[19] Schoenberg, I. J. “A Remark on M. M. Day's Characterization of Inner-product Spaces and a Conjecture of L.M. Blumenthal." Proceedings of the American Mathematical Society 3 (1952): 961-964. 